\title{
BRAIN TRAUMA
}

BRAIN INJURY AND SHAKING

The mechanism of brain injury in 24 infants with a diagnosis of shaken baby syndrome was investigated at the University of Iowa Hospitals and Clinics, Iowa City, IA. Half of the patients demonstrated signs of external head trauma. Intracranial injuries were indistinguishable in infants with and without evidence of extermal trauma. The mortality rate was the same in both groups. These findings indicated that shaking by itself is sufficient to cause severe or fatal intracranial injury and child abuse injuries associated with shaking may include direct trauma or only shaking. (Alexander R et al. Incidence of impact trauma with cranial injuries ascribed to shaking. AJDC June $1990 ; 144: 724-726$ ).

COMMENT. From an anatomical standpoint one might expect that shaking would cause damage to the brain in the distribution of the vertebral arteries. Brain stem and occipital hemorrhage or ischemia was not documented in this report however. Apparently, shaking may cause hemorrhage from various sites including the retina and the intracranial bridging veins and the subdural space. The data in this study suggests that shaking alone is sufficient to cause serious intracranial injury or death.

Brain injuries among infants, children, adolescents, and young adults are reviewed from the Department of Epidemiology School of Public Health, UCLA, Los Angeles, CA. (Kraus JF et al. AJDC June 1990; 144:684-691). The distribution of injuries by external cause varied with age. For infants, more than two-thirds of all brain injuries were from falls but only $8 \%$ were severe in nature. For preschool children, falls and motor vehicle accidents accounted for $51 \%$ and $22 \%$, respectively. For school aged children five to nine years old, injuries were equally divided among motor vehicles, falls, and sports and recreation-related activities $(31 \%, 31 \%$, and $32 \%$, respectively). Among adolescents aged 10-14 years, sports and recreationrelated activities accounted for $43 \%$ of all brain injuries but only $7 \%$ were serious. Among young adults, 55\% of injuries involved motor vehicles and one-third were serious.

\section{BRAIN INJURY AND DRONNING}

The epidemiology and clinical course of immersion brain injury of childhood drowning cases are reviewed from the University of California, Davis, Sacramento. In 1986 there were 2,122 childhood drownings in the United States. Children 0-4 years of age accounted for $36 \%$ of all deaths and most of these occurred in residential pools. Children with a seizure disorder are at increased risk for drowning with rates several times higher than expected. Alcohol use is a contributing factor among adolescents in 40-50\% of males. The outcome of drowning is largely determined by events occurring in the first ten 